\title{
Construction of Public Leisure PE Website Based on Struts Framework
}

\author{
Wenwei Jia, Yuan Gao, Minghai Yao \\ Department of Physical Education, Bohai University, \\ Jinzhou, 121013, China \\ College of Information Science and Technology, Bohai \\ University, Jinzhou, 121013, China
}

\begin{abstract}
The 21st century is the era of leisure, Leisure PE as a positive, healthy, civilized social life style in modern society, and promotes the harmonious development of man and society, and promotes the comprehensive and coordinated development of human beings. Only by strengthening the leisure PE propaganda, could promote the development of leisure PE. This paper constructs the public leisure PE website based on Struts framework. Firstly elaborated the definition of leisure PE and its importance to national health; secondly describes the three components of the Struts framework, including the model, view, and controller. and describes the working principle of Struts framework; Then the main function modules of leisure PE website has been designed, and with functional framework structure diagram; finally illustrate the implementation of leisure PE website specific modules based on Struts framework.
\end{abstract}

Keywords-leisure PE, website, Struts framework, functional framework, specific module

\section{INTRODUCTION}

Leisure $\mathrm{PE}$ is entertainment, fitness-oriented physical activity, it can not only exercise the body, but also can ease work and study pressure, appreciate a lot of fun. Leisure PE are the basis of mass PE. The development of leisure PE can not only promote the national healthy living, to meet the individual needs, establish a complete personality, correct national life attitude, cultivating national moral sentiments, but also enriches the cultural life of citizens, enhance national cultural standards, so that the national life quality get distillation and improve [1]. Leisure PE are a part of civilization, science, health lifestyle. Public lack of awareness on the concept of leisure PE, lack of scientific and reasonable guidance, increase leisure PE propaganda is an important way to the development of leisure PE. Use of leisure PE website build publicity and deepen public understanding of the leisure $\mathrm{PE}$ activities, leisure $\mathrm{PE}$ activities carried out on personal exercise, such as yoga, square dancing, rock climbing, swimming and so on. Deeply familiar with the importance of leisure PE, and enhance national fitness awareness, so leisure PE entered a good public opinion environment.

\section{STRUTS FRAMEWORK}

Struts are the general framework to realize MVC design pattern, which is composed of model, view, controller and xml. Struts architecture is shown Fig.1.

Model, in the Struts architecture, the model is divided into two parts: the internal state of the system and can change the operating of states (business logic). Internal state is usually represented by a group of ActionForm Bean. According to the design or application program complexity is different, which Bean can be self contained and has a continuous state, or only when the need to get data (from a database) [2].

View, the view is mainly established by the JSP, Struts contains extended custom tag library (TagLib), can simplify the process of creating fully internationalized user interface. The current tag library include: Bean Tags, HTML tags, Logic Tags, Nested Tags and Template Tags, and so on [1].

Controller, in the Struts architecture, the basic controller component is the instance Servlet in ActionServlet class, the actual use of the Servlet in the configuration file consists of a set of mapping (described by the ActionMapping class) are defined. For business logic operation is mainly composed of Action, ActionMapping, ActionForward, this several components coordination is completed, in which Action plays the implementor of real business logic, ActionMapping and ActionForward to specify the operation direction of different business logic or process [3].

Struts-config.xml is description file of a special information configuration related to the Struts. strutsconfig.xml configuration information is converted into a set of Action Mapping, and then be placed in Action Mappings container, controller with Action Mapping to the HTTP message user requests into application program action Action. Action Mapping specified request path, plan to process the request object and any services required other information. Action Mapping creates an Action object to handle the request. Once the Action objects to complete a task, it is in a JSP page through write the results directly response to user requests or it can make an application program flow to other places to do process [4]. 


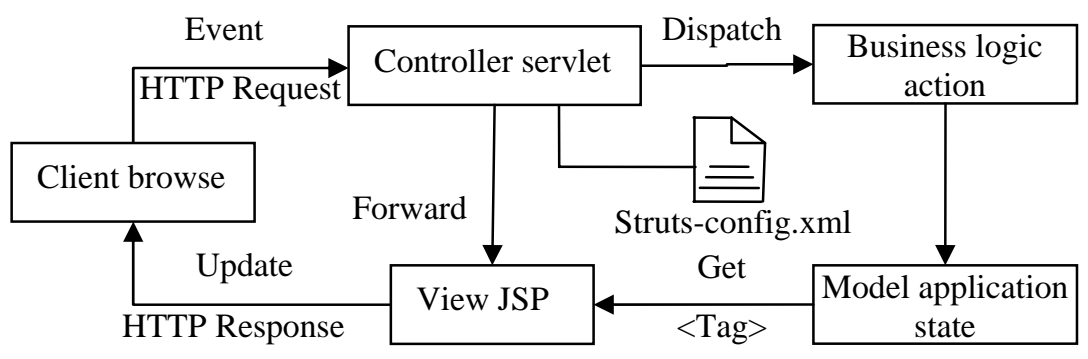

Figure 1. Struts architecture

\section{IIIFUNCTION MODULE DESIGN}

The functional framework structure for public leisure sports website based on Struts framework is shown in Fig.2. The main function modules are described as follows:

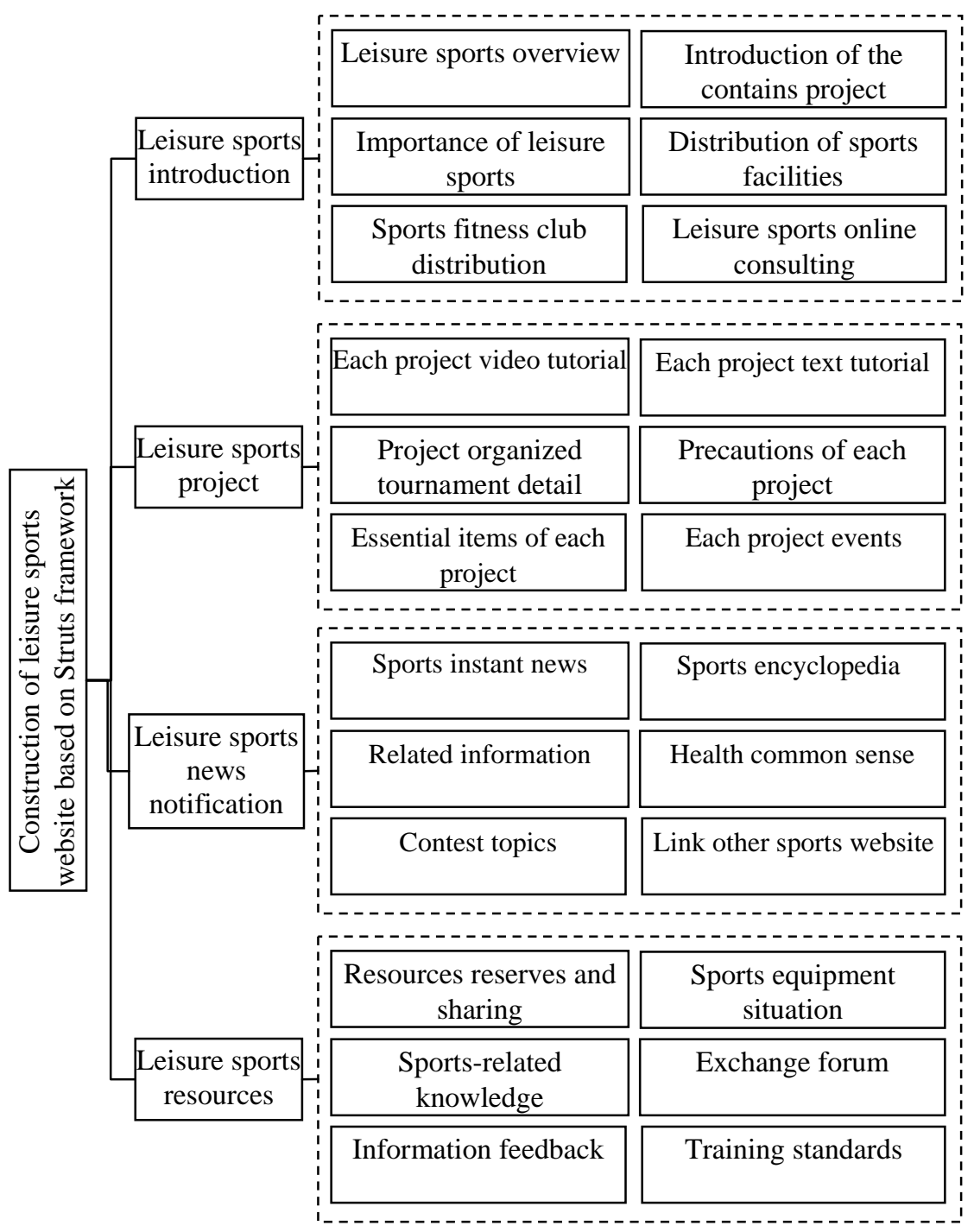

Figure 2. Functional framework of leisure sports website 
Leisure sports introduction, main function is to introduce the definition of leisure sports, the project contained exercises, broadcast gymnastics, aerobics, boating, group dance, picnic, outing, tai chi and so on. Strengthen people's awareness and emphasis on leisure time and leisure sports. Which also includes sports and fitness club is located, distribution, distribution of the gymnasium, to enable people to easily find the club, gymnasium, exercise the body uses sports facilities. There is also a sports consulting, do not understand the problem can be timely consultation.

Leisure sports project, mainly on a variety of leisure sports projects elaborate description, with video tutorials, text tutorials, can easily learn and download. Also include precautions of each project, safety tips, so that enable people to fitness in the circumstances of ensuring safety.

Leisure sports news notification refers to convey and disseminate a variety of information about the leisure sport, sports website aimed at the fastest, most innovative various sports information accurately convey to the people. Link is from the sports website links to other sports websites, is a measure of the convenience of users. Contest topics column is for the Olympic Games, National Games and other major events and timely reports, updates, to meet the public need for sports culture. The sports encyclopedia column is a highlight of sports website, to improve the visibility of sports websites, so that more people to pay attention to sports, joining fitness training team has a positive effect [5].

Resources reserve and sharing is one of importance of leisure sports website, website resource reserves, access to certain resources through the collection, collation or research and many other ways, and to share in the website, is conducive to the integration of resources and reserves and communication. Thereby creating a larger network storage space, Is an important channel for people to obtain sports knowledge, is conducive to the public use of cyber source for autonomous learning, and provide rich information resources for the national fitness cause and the sustainable development of social sports, its resources have a very large shared, resource reserves include sports related knowledge, training standards and so on. Information feedback refers to the message boards and other communication channels to collect views and information feedback in various aspects, this information includes mainly the website construction advice and content covers, related information and other aspects. By collecting on all aspects of information, in order to enrich the website content, and promote the website construction [6].

\section{SPECIFIC MODULE DESIGN}

Web applications using the Struts framework in Web application startup to load and initialize Action Servlet, Action Servlet reads the configuration information from the struts-config.xml file. By Action Servlet accept customer's request, and if you need ActionForm and ActionForm instance does not exist, create an ActionForm instance, to save form data submitted by the client to the ActionForm object, if you do not ActionForm, Action Servlet forwards the request to the appropriate Action. After Action by calling JavaBean/EJB complete various business logic, the results are returned to the Action Servlet, will eventually result returned by the Action Servlet to JSP, and generate dynamic pages, returned to the client [7].

The implementation of leisure sports website based to Struts framework, to query leisure sports information as an example[8]. Query leisure sports information to complete the function is when the user opens the website home page, information on the page will be selected, you can enter the desired content by searching or browsing a page on the desired content directly into the appropriate link, when input need contents, to carry out logic verification of form, logic verification is mainly to determine whether the format of the input information or information to meet the requirements, there is no problem when the logic verification, was transferred to the business verification of form. Concrete realization: First administrator uploaded leisure sports information resources, the user can be retrieved, and then browse them. Upload leisure sports information resources is the relevant information file into a database table, while browsing information is put on records in the database table to take out, stored in the target file, and then display the information page to make a JSP page displays form, the main contents and file names about relevant documents be take out, and made into hyperlinks form, as long as the user on the display page click on the hyperlink, it will automatically open this file in a IE browser. Information query program flow chart as shown in Fig.3. 


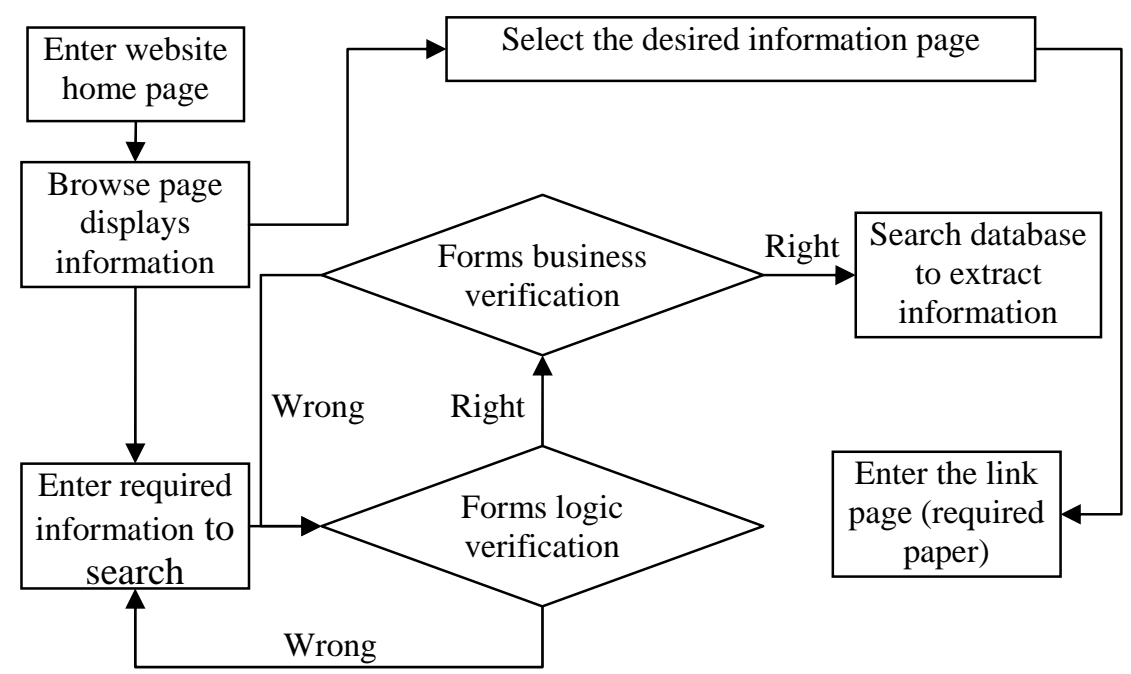

Figure 3. Query module flowchart

\section{CONCLUSIONS}

The leisure of public life for the rise and development of leisure sports has created unlimited growth space. Leisure sports have become an important content of public leisure era[9]. Leisure Sports is a conscious social sports, people participate in leisure sports, to reflect people care their health, quality of life[10]. Leisure sports in the future will become a leisure way of public life, through the construction of public leisure sports website, to increase the leisure sports publicity, pay attention to the important significance of leisure sports on people. Detailed description of the leisure sports, make people a clearer understanding of each item of leisure sports, and it is easy to learn, not only to promote public health life, meet people's diversified leisure need, but also promote the comprehensive development of people, and promote building a harmonious society also play a role.

\section{ACKNOWLEDGEMENTS}

This work is supported by social science fund project of Liaoning province (L13BTY004), Social Science Planning Foundation project of Liaoning province (No. L13BXW006).

\section{REFERENCES}

[1] F. Yang, H. X. Chen, "Research Culture Value in Improving People's Livelihood and Promote Social Harmony and Stability in Leisure Sports," Sport, vol. 6, no. 10, pp. 7-10, 2014.

[2] Baidu, "Struts framework," http://blog.csdn.net/fenglibing/article/details/1753481, 2014-9-30.

[3] BaiduBaike, "Struts ," http://baike.baidu.com/view/25603.htm, 2014-930 .

[4] X. Du, "Application in the Development of Large websites Based on Struts Framework of MVC Pattern," Chinese Journal of ICT in Education, vol. 14, no. 17, pp. 16-17, 2008.

[5] H. Z. Huang, "Study on the Status Quo of Sports Websites in Colleges in Fujian Province and Countermeasure," Xiamen University, 2009.

[6] X. F. Yue, "The Status and Strategy of Sport Portal Development of Undergraduate College in Henan Province," Journal of Anyang Institute of Technology, vol. 11, no. 14, pp. 93-96, 2012.

[7] L. Z. Bai, "Construction and Development Information Website Based on Struts Framework," Qinghai Electric Power, vol. 26, no. 1, pp. 5457, 2007.

[8] J. Bao, "Design Online Education Website Based on Struts Framework," Science and Technology Economy Market, vol. 23, no. 4, pp. 43-44, 2007.

[9] C. B. Xiao, "Discuss on Development of Public Life Leisure and Leisure Sports,” Sport, vol. 2, no. 1, pp. 134-135, 2010.

[10] J. Xu, "The Study on The Characters of The Residents' Leisure Sports Behaviors,” Journal of Guangzhou Physical Education Institute, vol. 26, no. $1, \quad$ pp. $97-100, \quad 2006$. 